\title{
A Cooperative Distributed System for Real-Time Route Guidance
}

\author{
Yaser E. Hawas \\ Civil and Environmental Engineering Department, UAE University, Al Ain, UAE \\ Email: y.hawas@uaeu.ac.ae
}

Received March 22, 2012; revised April 25, 2012; accepted May 20, 2012

\begin{abstract}
This paper describes a cooperative decentralized architecture for reactive real-time route guidance. The architecture is cooperative in the sense that it allows adjacent local controllers to exchange information regarding the traffic conditions in their territories. A set of local decision rules and associated heuristic functions to support the cooperative architecture are specified. A protocol governing the knowledge exchange among local adjacent controllers is developed. A simulation-assignment modeling framework is used for assessing the effectiveness of this cooperative architecture under various levels of controller knowledge and network traffic congestion. The cooperative decentralized system is tested under various scenarios of knowledge and cooperation and network traffic demand levels. The cooperative system is compared against the shortest path algorithm as a benchmark.
\end{abstract}

Keywords: Decentralized System; Real-Time Route Guidance; Local Controllers; Cooperative Systems; Micro-Scopic Simulation

\section{Introduction}

A common approach for route guidance envisions a central controller with capability to predict driver origindestination (O-D) trip desires, to optimally assign a path to each driver from origin to destination, as well as to re-route as warranted [1,2]. The reported limitations of centralized systems include the massive data processing and communication needs between the TMC and thousands of users at a time. Excessive computing, storage and communication capacities are required at the TMC. As a result, the TMC might be frequently overloaded [3]. Furthermore, such systems were reported to have high system operating costs [4].

In contrast, hierarchical distributed architectures provide for locally oriented real-time reactive strategies for vehicle routing that rely on limited available information $[5,6]$. In large-scale networks, the need for fast control action in response to local data inputs and perturbations strongly suggests use of distributed information and control structures. While distributed systems have been extensively exploited in areas such as telecommunications and computing network control, only recently have distributed systems been considered as a promising basis for route guidance in vehicular traffic networks.

Hawas and Mahmassani [2] developed a non-cooperative decentralized structure and a family of heuristic-based rules for reactive real-time route guidance. The premise of this decentralized structure is the ability to deal with varying degrees of information, spatially and temporally. In addition, unlike the centralized predictive approach, it does not require a priori knowledge (or prediction) of the time-dependent OD demand desires. This structure assumes a set of local controllers distributed over the network. Each local controller is responsible for providing reactive route guidance for vehicles in its territory. The controllers are non-cooperative in the sense that they do not exchange knowledge of the traffic states in their respective territories. Local decision rules that incorporate heuristic evaluation functions are specified, reflecting varying degrees of intelligence. The non-cooperative decentralized architecture has been shown to be computationally efficient, and fairly robust and effective under recurrent as well as incident situations [2].

The use of distributed multi-agent systems to improve dynamic route guidance and traffic management is reported in Adler et al. [7]. Inter vehicular communication (IVC) networks provide decentralized solutions for traffic management problems [8-11]. IVC networks are instantiations of mobile ad hoc networks, which have no fixed infrastructure and instead rely on ordinary nodes to perform network management functions.

There are several ITS projects based on IVC networks. FleetNet [9] uses an IVC network to improve the drivers and passengers' safety and comfort. VGrid [8] proposes solving vehicular traffic flow control problems autono- 
mously. TrafficView [10] defines a framework to disseminate and gather information about the vehicles based on IVC.

Hawas, Napeñas and Hamdouch [12] developed two algorithms for inter-vehicular communication (IVC)-based route guidance in a traffic network. Although the performance of such IVC-based algorithms is quite reasonable as compared to the centralized systems, there are still many challenges such as the rapid topology changes, the frequent fragmentations and the small effective network diameter. Because of the high relative speed of vehicles, the IVC network experiences very rapid changes in topology. Also, due to the low deployment of vehicles having IVC, the IVC network is subject to frequent fragmentation. Finally, because of the poor connectivity, the effective network diameter is usually small. These aspects impose restrictions if deployed via IVC technologies. For instance, one should compromise the extra effectiveness of having wider ranges of communication against the possible degradation in performance due to poor communication.

Bearing in mind the massive data processing and high operational cost associated with the centralized systems, the instability and communication constraints associated with the IVC-based systems, this paper seeks to provide improvement to the earlier work of Hawas and Mahmassni [2]. The improvement is intended to resolve the reported cycling problems commonly encountered in the typical pure distributed systems. The improvement is sought through allowing for information exchange (or cooperation) among the various decentralized controllers. In a sense, we investigate the possibility of using intercontroller communication for exchanging knowledge regarding the traffic conditions in their respective territories. Such improvement is thought of as a way to overcome the limitations of the rapid topology changes, the frequent fragmentation and poor communication associated with the IVC-based systems, as well as the limitations of the heavy processing and cost of the centralized systems. The information exchange would enrich the knowledge base of any individual controller, and potentially improve the quality of control by providing the opportunity to utilize higher degrees of intelligence to improve the specification of the heuristic evaluation functions underlying the local decision rules. This new system shall be denoted in this paper by the cooperative decentralized system.

The paper is organized in five sections. Section 2 reviews the detail the decentralized system structure, assumptions, and rule specifications. Then details of the rationale, and the essential modifications to the rule specifications and heuristics to support the cooperative decentralized scheme are presented. Section 3 discusses the off-line simulation experimental design for the de- centralized schemes for vehicle routing. Section 4 provides comparative estimates of the performance of both decentralized route guidance non-cooperative and cooperative algorithms, with particular emphasis on the improvement in performance obtained by allowing knowledge sharing among the local controllers. Section 5 provides some concluding comments.

\section{Decentralized Route Guidance Strategies}

Hawas and Mahmassani [2] presented a distributed architecture that provides for locally oriented real-time strategies for reactive route guidance. This decentralized architecture has the ability to deal with situations where only limited information is available to the controllers. The decentralized architecture envisioned a set of local controllers distributed over the network, where every controller can extract only limited "raw" information (speed, concentration, etc.) from detectors, and utilizes this information in conjunction with local decision rules to guide vehicles within its territory to their individual destinations. The local controllers are specific hardware units that may be located at the level of the network intersection; the decentralization level could be coarser or finer depending on the available hardware, the level of investment and the desired accuracy. Figure 1 illustrates the spatial extent of the area from which the controller at node $i$ extracts information. The size of information extracted by a single controller (denoted by the knowledge level, $K$ ) refers to the number of downstream links, from which traffic measurements can be measured and utilized in making the routing decisions. Figure 1 shows an example of a local controller with $K$ equals $1,2,3$ and 4, respectively.

Local decision rules use available partial information and heuristics to evaluate alternative subpaths emanating from the decision node towards the destination, and assign vehicles at that node to the $\operatorname{link}(\mathrm{s})$ immediately downstream. The vehicle could be assigned to only one link, or multiple successive links within the local area. At the end node of the assigned portion, the vehicle reaches

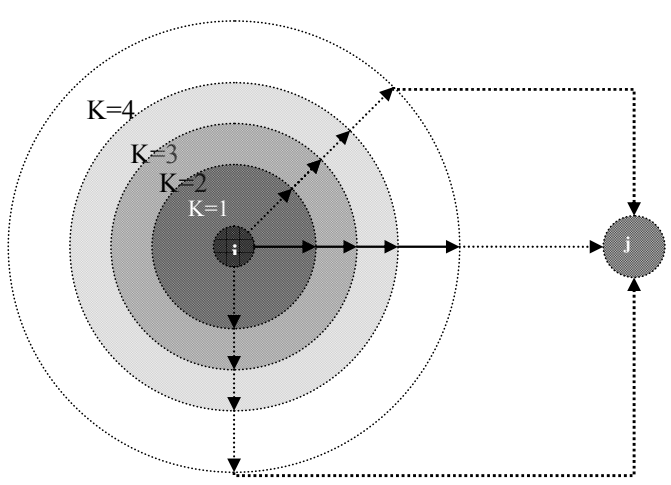

Figure 1. Controller $i$ with various knowledge levels. 
another local controller, where a new assignment is instructed. A subpath $(i, j, m, K)$ denotes the first $(K)$ links of path $(m)$ from the decision node $(i)$ to destination $(j)$. Assignment decisions are reached after evaluating the various alternative subpaths in the local area. The controller uses the current state in the local area and the anticipated state outside the local area to evaluate the alternative subpaths. The subpath evaluation is analogous to that of the A* graph search algorithm, which uses heuristic information to decide which node to scan first. The distinctive feature of the $A^{*}$ algorithm is the definition of the evaluation function, $F$, which has two components: the cost of reaching the node from the start node, $G$, and the cost of reaching the goal from the node, $H$. The node to expand is the one for which $F=G+H$ is minimum [13].

Consider a vehicle $v$ going from origin node $O(v)$ to destination node $D(v), v=1, \cdots, V$. Let $t$ denote the time at which $v$ is about to cross a given controller $i$ in its way to $D(v)$. The problem is to assign vehicle $v$ to an outgoing link (or links) emanating from $i$. Upon reaching the downstream end node of the assigned link(s), the vehicle is similarly assigned to another outgoing $\operatorname{link}(\mathrm{s})$, and so on until $v$ reaches $D(v)$.

The type of local assignment rule considered here selects, at node $i$, a $K$-link subpath on the basis of current knowledge of travel time, distance, and concentration along all the possible $K$-link subpaths (emanating from node $i$ ). A penalty function $G_{i, j, \ell}^{t}$ is defined to evaluate the $K$ links on subpath $\ell$, using their current state.

The anticipated cost of non-local portion of the vehicle's trip (from the end of the subpath to the vehicle's destination) is estimated using a heuristic penalty function $H_{i, j, \ell}^{t}$. The total penalty, given by $F_{i, j, \ell}^{t}=G_{i, j, \ell}^{t}+H_{i, j, \ell}^{t}$, provides the basis for evaluating the alternative subpaths. The specification of the heuristic function may reflect varying degrees of "knowledge", with varying corresponding effort in terms of computation, data acquisition, data processing and/or prediction. Figure 2 shows an example of a subpath of nodes from controller $i$ to node $j$, and illustrates the functions $G$ and $H$.

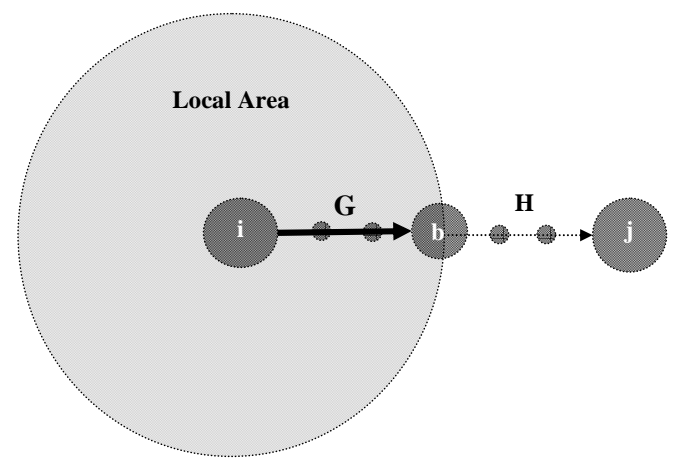

Figure 2. The performance functions $G$ and $H ; F=G+H$.
Two different structures are discussed afterwards. The first is a non-cooperative structure where controllers work independently. This is denoted by the "pure" decentralized structure. Every controller is assumed to have access to information and traffic measurements from its own territory, and share no information with adjacent controllers. The second is a cooperative structure, where controllers are envisioned to share useful information with adjacent controllers regarding the traffic states of their own territories. This cooperative scheme provides for the mechanism to enrich the knowledge base of individual controllers.

\subsection{Non-Cooperative Decentralized Route Guidance}

Hawas and Mahmassani (1996) presented the generalized rules that can be used to distribute vehicles among several subpaths using a logit splitting model. A generalized subpath penalty function was developed. It comprised local state variables (travel-time and concentration), and non-local variables (anticipated travel time). A penalty coefficient was introduced and associated with each state variable to reflect the variable's relative impact on the subpath total penalty. The penalty function comprised three sets of state variables: 1) local variables to measure congestion (average weighted concentration); 2) local and non-local variables to measure traveling distance; and 3) local and non-local variables to measure travel time.

The local portion of the penalty function $G_{i, j, \ell}^{t}$ was specified as follows:

$$
G_{i, j, \ell}^{t}=\beta_{T}^{L} \cdot T_{i, j, \ell}^{L, t}+\beta_{K}^{L} \cdot K_{i, j, \ell}^{L, t}+\beta_{S}^{L} \cdot S_{i, j, \ell}^{L}
$$

where $\beta_{T}^{L}, \beta_{K}^{L}$, and $\beta_{S}^{L}$ are the penalty coefficients of the current local travel time along subpath $\ell$ at time $t$, $T_{i, j, \ell}^{L, t}$, the current average concentration, $K_{i, j, \ell}^{L, t}$, and the local traveled distance, $S_{i, j, \ell}^{L}$, respectively. $T_{i, j, \ell}^{L, t}$ is the sum of the link travel times along subpath $\ell$ at time $t$; $S_{i, j, \ell}^{L}$ is the sum of link distances along $\ell$, and is given by:

$$
S_{i, j, \ell}^{L}=\sum_{a \in \ell} S(a)
$$

where $S(a)$ is the length of link $a$.

In Equation (1), $K_{i, j, \ell}^{L, t}$ denotes the average concentration along the subpath, as an indicator of the local congestion level. The current concentration also reflects the average number of vehicles to be affected by the assignment. If the coefficient $\beta_{K}^{L}$ of this variable is interpreted as the average additional (marginal) cost imposed on the subpath vehicles, then $\beta_{K}^{L} \cdot K_{i, j, \ell}^{L, t}$ would indicate the additional delay (per unit distance) incurred by the subpath vehicles. The effect on the subpath vehicles diminishes gradually as vehicles get further from the deci- 
sion node. Link concentrations are weighted inversely to account for the spatially diminishing effect, as follows:

$$
K_{i, j, \ell}^{L, t}=\frac{\sum_{a \in \ell} \frac{C^{t, a}}{D(a)}}{\sum_{a \in \ell} \frac{1}{D(a)}}
$$

where $C^{t, a}$ is the current concentration (vehicles/unit distance) of link $a$ at time $t$, and $D(a)$ is the depth of link $a$ with respect to the decision node, $i$.

The specification of the non-local portion of the evaluation function, $H_{i, j, \ell}^{t}$ is given by:

$$
H_{i, j, \ell}^{t}=\beta_{\tilde{T}}^{N L} \cdot \tilde{T}_{i, j, \ell}^{N L, t}+\beta_{\tilde{S}}^{N L} \cdot \tilde{S}_{i, j, \ell}^{N L}
$$

where, $\beta_{\tilde{T}}^{N L}$ and $\beta_{\tilde{S}}^{N L}$ are the penalty coefficients of the non-local anticipated travel-time, $\tilde{T}_{i, j, \ell}^{N L, t}$, and the nonlocal anticipated traveled distance, $\tilde{S}_{i, j, \ell}^{N L}$, respectively. The state variable $\tilde{T}_{i, j, \ell}^{N L, t}$ is an approximation of the anticipated non-local travel time from the end of subpath $\ell$ to destination $j ; \tilde{T}_{i, j, \ell}^{N L, t}$ can be calculated by extrapolating the local prevailing travel time, from historical information, or it may be replaced by corresponding information exchanged from the adjacent controllers under the cooperative scheme as will be discussed later.

Because the actual path to be followed beyond the local area boundaries is not known a priori, heuristics are used to obtain coarse estimates of $\tilde{S}_{i, j, \ell}^{N L}$, and $\tilde{T}_{i, j, \ell}^{N L, t}$. While recognizing that the specification could reflect varying degrees of intelligence, and involve correspondingly varying amount of computational processing; our intent was to test very simple specifications that did not require any network path computations for the non-local portion.

A particular specification of $\tilde{T}_{i, j, \ell}^{N L, t}$ is given by:

$$
\tilde{T}_{i, j, \ell}^{N L, t}=\frac{T_{i, j, \ell}^{L, t}}{S_{i, j, \ell}} \cdot \tilde{S}_{i, j, \ell}^{N L}
$$

The non-local travel distance, $\tilde{S}_{i, j, \ell}^{N L}$, is calculated by substituting the Cartesian coordinates of the subpath end node $b n$ at the boundary and those of the destination node $j$ into a weighted sum of both "Manhattan" (rightangle) and "Euclidean" distances between the two points (Equations (6) and (7)).

$$
\begin{aligned}
\tilde{S}_{i, j, \ell}^{N L}= & {\left[W_{M}\left\{\left(x_{j-} x_{b n}\right)+\left(y_{j-} y_{b n}\right)\right\}\right.} \\
& \left.+W_{E}\left\{\left(x_{j-} x_{b n}\right)^{2}+\left(y_{j-} y_{b n}\right)^{2}\right\}^{0.5}\right] \\
W_{M} \geq & 0, W_{E} \geq 0 ; \text { and } W_{M}+W_{E}=1
\end{aligned}
$$

The parameters $W_{M}$ and $W_{E}$ are selected according to the general network topology. For ideal grid networks the distance between any two nodes is the Manhattan distance, and $W_{M}=1$ with $W_{E}=0$.
This rule distributes vehicles among several feasible subpaths using a splitting model, which allocates more vehicles to least cost subpaths, and fewer vehicles to subpaths with worse performance, in an attempt to equalize the performance on all emanating feasible subpaths. A subpath $\ell$ belongs to the subset of feasible subpaths, $\Gamma(i) \subseteq F S(i) \quad$ (where $F S(i)$ is the set of all subpaths emanating from $i$ if the following condition applies:

$$
F_{i, j, \ell}^{t}<F_{i, j, \ell^{*}}^{t}(1+\delta), \quad \delta \geq 0
$$

where $F_{i, j, \ell^{*}}^{t}$ is the minimum value of $F_{i, j, \ell}^{t}$

$\forall \ell \in F S(i)$. For $\delta$ equals $0, \Gamma(i)$ includes only the best subpath, $\ell^{*}$, and this rule operates as all-or-nothing assignment. If $\delta \rightarrow \infty$, then $\Gamma(i) \equiv F S(i)$.

The fraction of vehicles assigned to a feasible subpath $\ell$, is inversely proportional to the penalty value, $F_{i, j, \ell}^{t}$. Several functional forms could be used for the splitting rule. A standard logit form was used as follows:

$$
p_{i, j, \ell}^{t}=\frac{e^{\theta\left(F_{i, j, \ell}^{t}-F_{i, j, \ell^{*}}^{t}\right)}}{\sum_{f} e^{\theta\left(F_{i, j, \ell}^{t}-F_{i, j, \ell^{*}}^{t}\right)}}, f \in \Gamma(i), \forall \ell \in \Gamma(i), i, j, t
$$

The model allocates vehicles to any subpath based on the difference between the subpath performance, $F_{i, j, \ell}^{t}$, and the performance of the best subpath, $F_{i, j, \ell^{*}}^{t}$. The parameter $\theta$ is the dispersion factor and its value is typically negative.

The above rule specification required the calibration of the time-dependent penalty coefficients. A simulationbased optimization procedure that employs a variant of the well-known Hill Climbing Technique was used. The penalty coefficients were calibrated so as to optimize the overall network performance over the analysis period T.

\subsection{Cooperative Decentralized Route Guidance}

A mathematical optimal control formulation is developed for the derivation of the optimal specification of the heuristic function $G_{i, j, \ell}^{t}$, in a simplified network. The penalty function is specified as the approximate current marginal travel time along the subpath. This can be expressed as:

$$
G_{i, j, \ell}^{t}=T_{i, j, \ell}^{L, t}+M \cdot K_{i, j, \ell}^{L, t} \cdot S_{i, j, \ell}^{L, t}
$$

The term $\left(K_{i, j, \ell}^{L, t} \cdot S_{i, j, \ell}^{L, t}\right)$ expresses the total number of vehicles along subpath $\ell$. The coefficient $M$ is the average marginal effect of the added vehicle at $i$ on any of the $\left(K_{i, j, \ell}^{L, t} \cdot S_{i, j, \ell}^{L, t}\right)$ vehicles. The heuristic function can be specified as:

$$
H_{i, j, \ell}^{t}=\tilde{T}_{i, j, \ell}^{N L, t}
$$


Exchanging knowledge among local controllers is intended to reduce the influence of errors introduced by the heuristic function $H_{i, j, \ell}^{t}$ in calculating the non-local variables. One might utilize abstract or processed information from neighboring controllers to improve the heuristic function specification. While incorporating a higher knowledge level might increase the computational burden significantly, combining abstract information (from adjacent controllers) increases the knowledge at negligible cost. The cooperative control scheme envisions a set of controllers connected through a two-way communication system. The exchanged knowledge is incorporated in the heuristic function specification $H_{i, j, \ell}^{t}$ to better anticipate traffic conditions in the non-local portion. Figure 3 outlines a schematic representation of the cooperative distributed system.

Different cooperative schemes may be defined depending on the relative locations of the controllers that may exchange knowledge, the information to be exchanged, and the specification of the heuristic function $H_{i, j, \ell}^{t}$.

\subsection{Cooperative Decentralized System Structure}

A two-way communication system is envisioned to allow the controller at $i$ to receive knowledge from those controllers that reside at its boundary nodes. The territories of any two communicating controllers will overlap and share an area of depth $K$ (if both controllers have the same knowledge level $K$ ). Figure 4 shows a simplified network (with $K=2$ ) where controllers share information. As shown, controller $i$ receives information from controller $c$ and all other depth- $K$ controllers to improve the specification of the heuristic function $H_{i, j, \ell}^{t}$. Assume that $\ell$ is a subpath from $i$ to $j$, where $c$ is the boundary node of $\ell$. Assume subpath $f^{*}\left(f^{*}=[c, m, d]\right)$, as shown in Figure 4, is the least travel time subpath from $c$ to $j$ at time $t$.

Controller $i$ receives the following information items from controller $c$ at time $t$ :

1) Average concentration in the local area governed by controller $c$.

2) Estimated travel time estimate from $c$ to the destination node $j$ along the least travel time subpath, $f^{*}$. The decision controller at $i$ utilizes this information only if $c$ is closer to the destination node, $j$.

3) Distance from $c$ to the destination node $j$ along the least travel time subpath, $f^{*}$. The decision controller at $i$ utilizes this information only if $c$ is closer to the destination node, $j$.

This information is used by controller $i$ to improve the specification of the heuristic function $H_{i, j, \ell}^{t}$. The heuristic function $H_{i, j, \ell}^{t}$ (Equation (4)) is modified by introducing a new variable as well as a penalty factor, $\beta_{K}^{E}$ of the exchanged concentration from $c$, as will be shown later. Travel time information from $c$ is used to replace the term $\tilde{T}_{i, j, \ell}^{N L, t}$ in $H_{i, j, \ell}^{t}$ (Equation (4)) by $T_{c, j, f^{*}}^{L, t-1}+\tilde{T}_{c, j, f^{*}}^{N L, t}$,

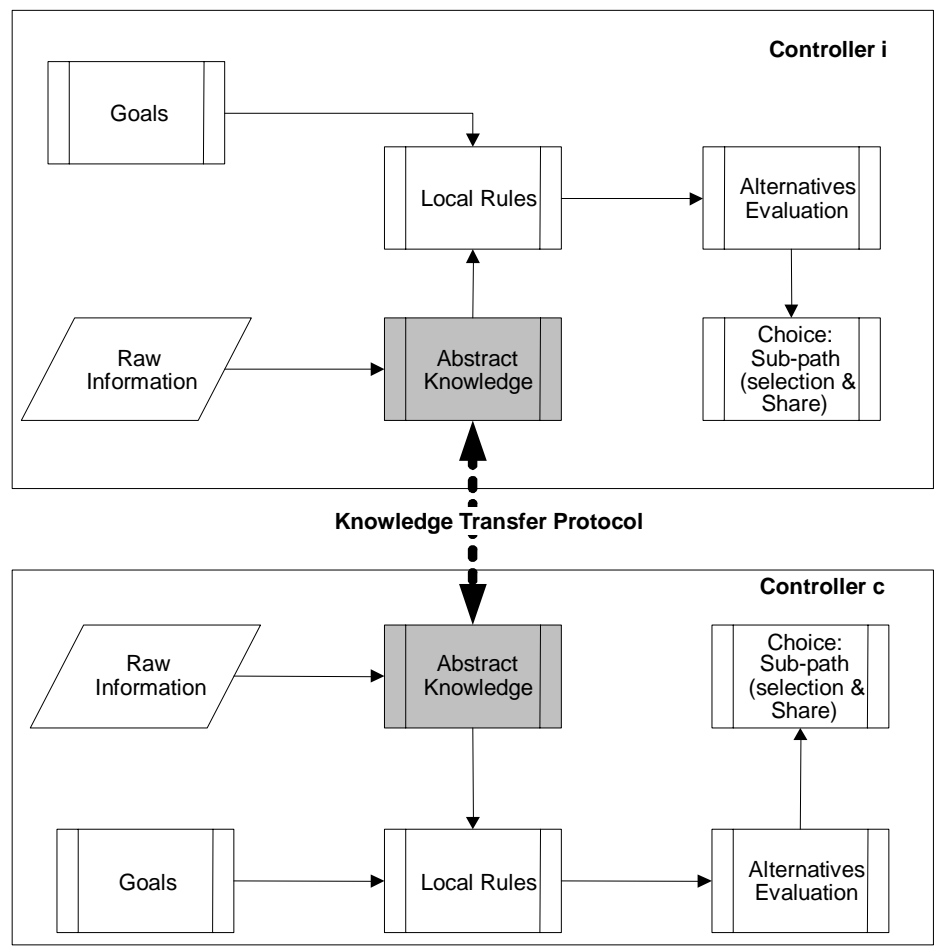

Figure 3. Structure of the cooperative decentralized system. 


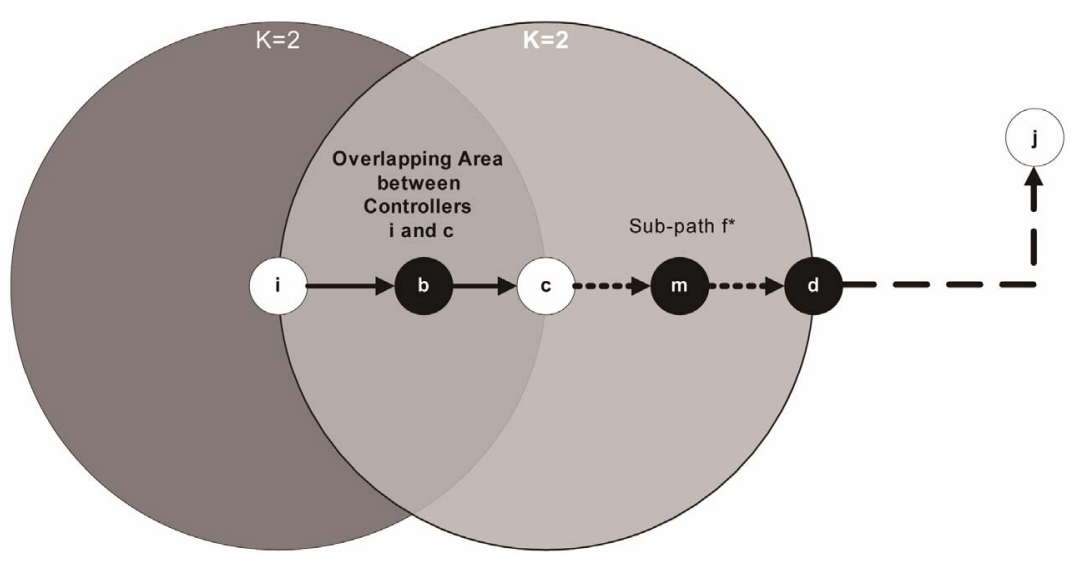

Figure 4. Information exchange with spatial propagation.

where $T_{c, j, f^{*}}^{L, t-1}$ is the prevailing travel time along subpath $f^{*}$ at time $t^{c, j, f^{*}}-1$, and $\tilde{T}_{c, j, t f^{N *}}^{N L,-1}$ denotes the "anticipated" non-local travel time from the boundary node of subpath $f^{*}$ (node $d$ as shown in Figure 4) to $j$, at time $t-1$. Similarly, the distance information is used to replace $\tilde{S}_{i, j, \ell}^{N L}$ (in Equation (4)) by $S_{c, j, f^{*}}^{L}+\tilde{S}_{c, j, f^{*}}^{N L}$, where, $S_{c, j, f^{*}}^{L}$ is the prevailing distance traveled along subpath $f^{*}$, and $\tilde{S}_{c, j, f^{*}}^{N L}$ denotes the "anticipated" non-local distance from the boundary node of subpath $f^{*}$ (node $d$ ) to $j$.

\subsection{Knowledge Exchange Protocol}

Figure 5 shows a controller $i$ exchanging knowledge with all the boundary controllers $[a, b, c, e, f, g, k]$. In the figure, concentration information is exchanged between $i$ and all depth- $K$ controllers. Travel time and distance information are utilized from three boundary controllers only $[a, b, c]$. Denote by, $K_{i, j, \ell}^{E, t}$, the concentration received by $i$ (from the controller at the end node of $\ell, c$ ) at time interval t. $\beta_{K}^{E}$ is the penalty coefficient of the concentration term, $K_{i, j, \ell}^{E, t}$. As indicated previously, this information is processed by controller $\mathrm{c}$, at interval $t-1$. The heuristic function $H_{i, j, \ell}^{t}$ (for subpath $\ell$ from controller $i$ at time $t$ ) is now re-specified as:

$$
\begin{aligned}
H_{i, j, \ell}^{t} & =\beta_{\tilde{T}}^{N L}\left[x \cdot \tilde{T}_{i, j, \ell}^{N L, t}+y \cdot\left(T_{c, j, f^{*}}^{L, t-1}+\tilde{T}_{c, j, f^{*}}^{N L, t-1}\right)\right] \\
& +\beta_{\tilde{S}}^{N L}\left[x \cdot \tilde{S}_{i, j, \ell}^{N L}+y \cdot\left(S_{c, j, f^{*}}^{L}+\tilde{S}_{c, j, f^{*}}^{N L}\right)\right] \\
& +\beta_{K}^{E} \cdot K_{i, j, \ell}^{E, t}
\end{aligned}
$$

where, $x$ and $y$ are binary indicators. $x=0$ and $y=1$ if the Manhattan distance from $c$ to $j$ is greater than the Manhattan distance from $i$ to $j$, otherwise, $x=1$ and $y=$ 0 . In other words, the travel time and distance information are utilized only if the relaying controller, $c$, is closer to the destination node, $j$, than the receiving controller, $i$. The above specification replaces the heuristic function non-cooperative specification (Equation (4)).

The same protocol is also applied to the specification derived using the optimal control theory. The exchanged knowledge is utilized to enhance the heuristic function (Equation (10)) by adding a term that captures the marginal cost along subpath, $f^{*}$. The travel time information is also utilized according to the binary values of $x$ and $y$, as indicated above. The specification of the heuristic function becomes:

$$
\begin{aligned}
& H_{i, j, \ell}^{t}=x \cdot \tilde{T}_{i, j, \ell}^{L, t}+y \cdot\left(T_{c, j, f^{*}}^{L, t-1}+\tilde{T}_{c, j, f^{*}}^{N L, t-1}\right) \\
& +M_{2}\left(K_{i, j, \ell}^{E, t} \cdot S_{c, j, f^{*}}^{L}\right)
\end{aligned}
$$

where $M_{2}$ is the coefficient of the marginal cost of $f^{*}$. Note that if the concentration coefficient, $M_{2}$, is forced to a value of 0 , it will represent the case that concentration information is not exchanged among controllers. Various operational modes can be tested based on the values of $x$, $y$, and $M_{2}$ :

1) FCD refers to the fully cooperative decentralized system where individual controllers may have full access to knowledge processed by adjacent depth- $K$ controllers, i.e. $x$ and $y$ values are set according to the controllers locations with respect to the destination node.

2) $P C D$ refers to the partially cooperative distributed system where controllers may only access part of the adjacent controllers' information (the concentration is not shared among controllers), i.e. $x$ and $y$ values are set according to the controllers locations with respect to the destination node, but $M_{2}$ is set to 0 .

3) NCD which refers to the non-cooperative scheme that permits no information exchange among controllers, i.e. $x=1, y=0, M_{2}=0$.

\section{Experimental Design}

Simulation experiments are conducted to assess the effectiveness of the decentralized reactive approach, with particular emphasis on the cooperative scheme. The latter is tested under various scenarios of network congestion, and knowledge levels. The experiments address the following aspects: 


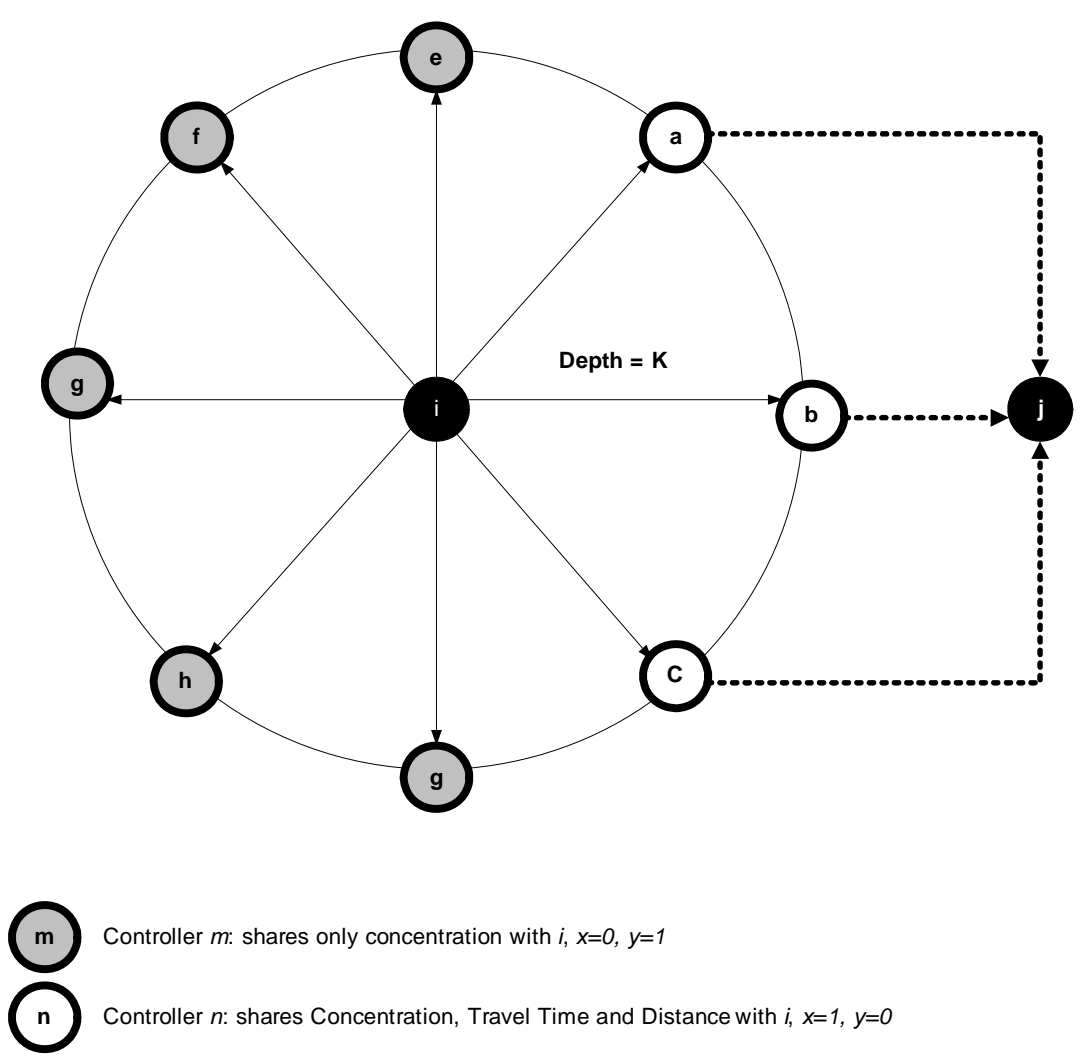

Figure 5. Type of Information utilized from depth-K controllers.

- The performance of the decentralized scheme is examined under various network congestion and knowledge levels.

- The cooperative scheme is evaluated against the above scenarios, for various network congestion levels, knowledge levels, and the three operational modes discussed earlier ( $F C D, P C D$ and NCD). By comparing the network performance under both non-cooperative and cooperative decentralized schemes, the marginal effect of the knowledge exchange can be assessed under the various knowledge and congestion levels. Under the FCD mode, travel time, distance and concentration information could be exchanged. The PCD mode allows only travel time and distance to be exchanged. Exchanged information replace the heuristic estimates as warranted by the protocol presented earlier. The difference in performance between the NCD, PCD, and FCD modes could be attributed to the effect of exchanged information in the latter modes. Similarly, the difference between the FCD and $P C D$ modes is attributed to the effect of the additional information (namely, $K_{i, j, \ell}^{E, t}$, average concentration).

\section{Simulation Modeling}

To test the overall network performance under the con- trol of both centralized and decentralized architectures, an analysis period of $T$ (60 minutes) is defined. The solutions obtained by both types of control is simulated over the analysis period using i-sim-s simulator (Hawas 2007, Hawas and Abdel Hameed, 2009), and estimates of the overall network travel time are obtained for comparative analysis of effectiveness.

One hypothetical network is coded for testing. The hypothetical network links are bi-directional with same posted (free-flow) speed. The use of hypothetical networks for the assessment of the algorithms is quite adequate as it enables testing various scenarios of network size, link lengths, speeds, etc. The hypothetical testing network as shown in Figure 6 has 49 nodes, 14 origins and 24 destinations. All intersections are operated with pre-timed controllers. The network is tested under various conditions of network congestions (demand levels) as shown in Table 1.

The experimental setup accounts for the effect of the operational modes (column one), variations in the knowledge levels (second column), and the variation in the OD demand pattern (third column). The first $\left(1^{\text {st }}\right)$ column shows the operational modes (FCD, PCD, and NCD). Each mode is tested with three knowledge levels, $K(1,3$, 5). Each operational mode (and knowledge levels) is tested with three demand scenarios. As the table indicates 


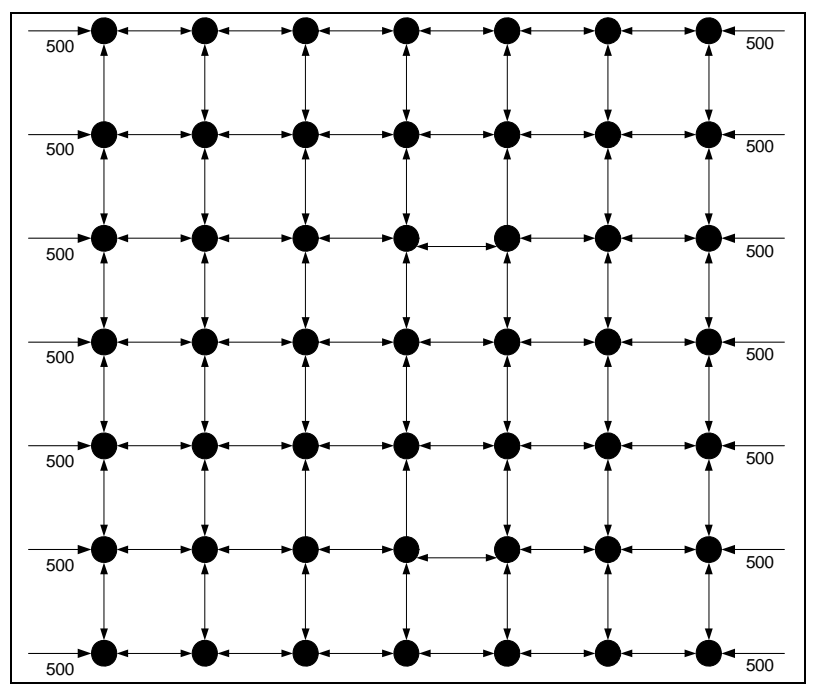

(a)

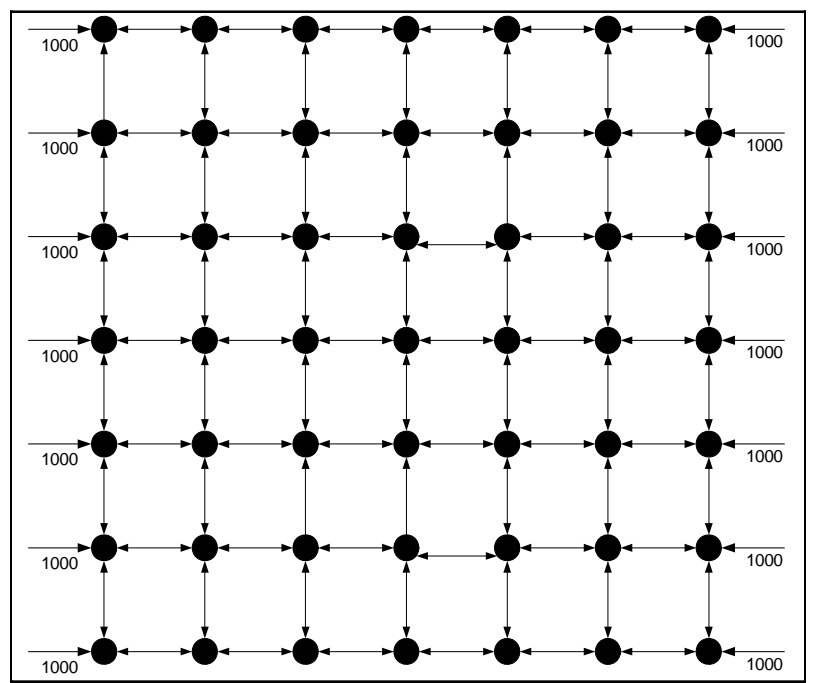

(b)

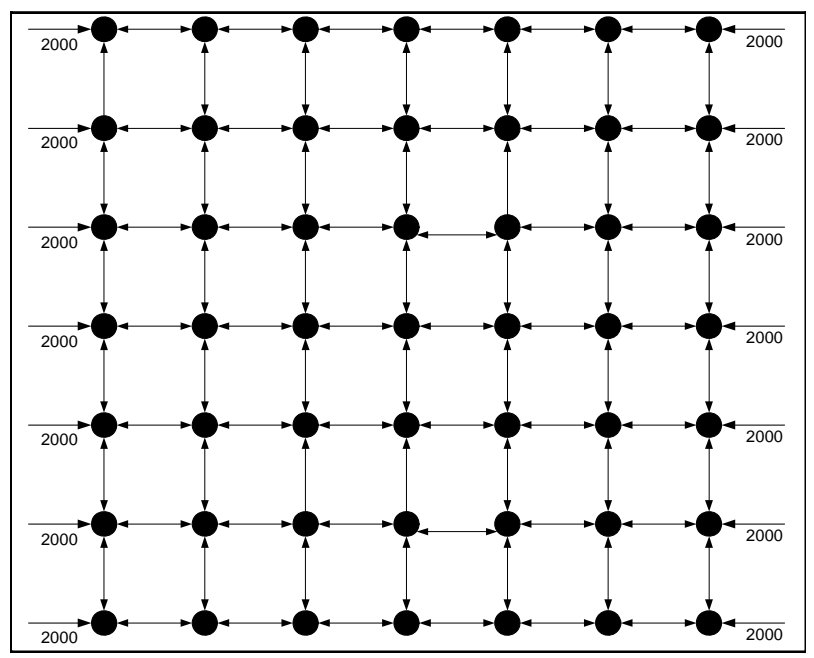

(c)

Figure 6. Demand patterns of the test network: (a) Low; (b) Medium and (c) High intensities.
Table 1. Testing scenarios.

\begin{tabular}{|c|c|c|}
\hline $\begin{array}{l}\text { Operational } \\
\text { Mode }\end{array}$ & $\begin{array}{l}\text { Knowledge } \\
\text { Level }(K)\end{array}$ & $\begin{array}{l}\text { Source Volume } \\
\quad(\mathrm{veh} / \mathrm{hr})\end{array}$ \\
\hline \multirow{9}{*}{$\begin{array}{c}F C D \\
\text { (Fully Cooperative) }\end{array}$} & \multirow{3}{*}{1} & 500 \\
\hline & & 1000 \\
\hline & & 2000 \\
\hline & \multirow{4}{*}{3} & 500 \\
\hline & & 1000 \\
\hline & & 2000 \\
\hline & & 500 \\
\hline & \multirow[t]{3}{*}{5} & 1000 \\
\hline & & 2000 \\
\hline \multirow{9}{*}{$\begin{array}{c}P C D \\
\text { (Partially Cooperative) }\end{array}$} & & 500 \\
\hline & \multirow[t]{2}{*}{1} & 1000 \\
\hline & & 2000 \\
\hline & \multirow{3}{*}{3} & 500 \\
\hline & & 1000 \\
\hline & & 2000 \\
\hline & \multirow{3}{*}{5} & 500 \\
\hline & & 1000 \\
\hline & & 2000 \\
\hline \multirow{9}{*}{$\begin{array}{c}N C D \\
\text { (Not-Cooperative) }\end{array}$} & \multirow{3}{*}{1} & 500 \\
\hline & & 1000 \\
\hline & & 2000 \\
\hline & \multirow{3}{*}{3} & 500 \\
\hline & & 1000 \\
\hline & & 2000 \\
\hline & \multirow{3}{*}{5} & 500 \\
\hline & & 1000 \\
\hline & & 2000 \\
\hline \multirow{3}{*}{$\begin{array}{c}\text { Benchmark: SPA } \\
\text { (Shortest Path Algorithm) }\end{array}$} & & 500 \\
\hline & & 1000 \\
\hline & & 2000 \\
\hline
\end{tabular}

the FCD is tested twenty seven (27) times.

The "network demand" scenarios are aimed at studying the effect of the operational mode, and the knowledge level under various network demand volumes. As such, all the network demand experiments are carried out for the same network topography (link length is kept fixed; $500 \mathrm{~m})$ and same link speed $(80 \mathrm{~km} / \mathrm{hr})$.

The traffic loading intensity varies from low (source volume of $500 \mathrm{veh} / \mathrm{hr}$ ), medium (source volume of 1000 $\mathrm{veh} / \mathrm{hr}$ ) and high (source volume of $2000 \mathrm{veh} / \mathrm{hr}$ ). In all tested scenarios, the source volumes are equally distributed among all possible destinations.

The Shortest Path Algorithm (SPA) is used as the benchmark in all the above set of experiments. That is, 
the resulting network performance (the overall network travel time) is compared with the corresponding value if a real-time SPA is deployed.

\section{Experimental Results}

Experiments with the various operational modes were conducted using various network demand levels (500, 1000 and $2000 \mathrm{veh} / \mathrm{hr}$ ) and knowledge levels (1,3 and 5), and results are summarized in Table 2 and Figure 7.

At very low knowledge levels, vehicles may cycle in the network, and this may lead to high degradation in performance compared to the benchmark. The results indicate that the marginal improvement in performance is higher for low knowledge levels.

The decentralized scheme is tested under three knowledge levels (1,3 and 5), demand levels of 500, 1000 and

Table 2. Cooperative decentralized system performance.

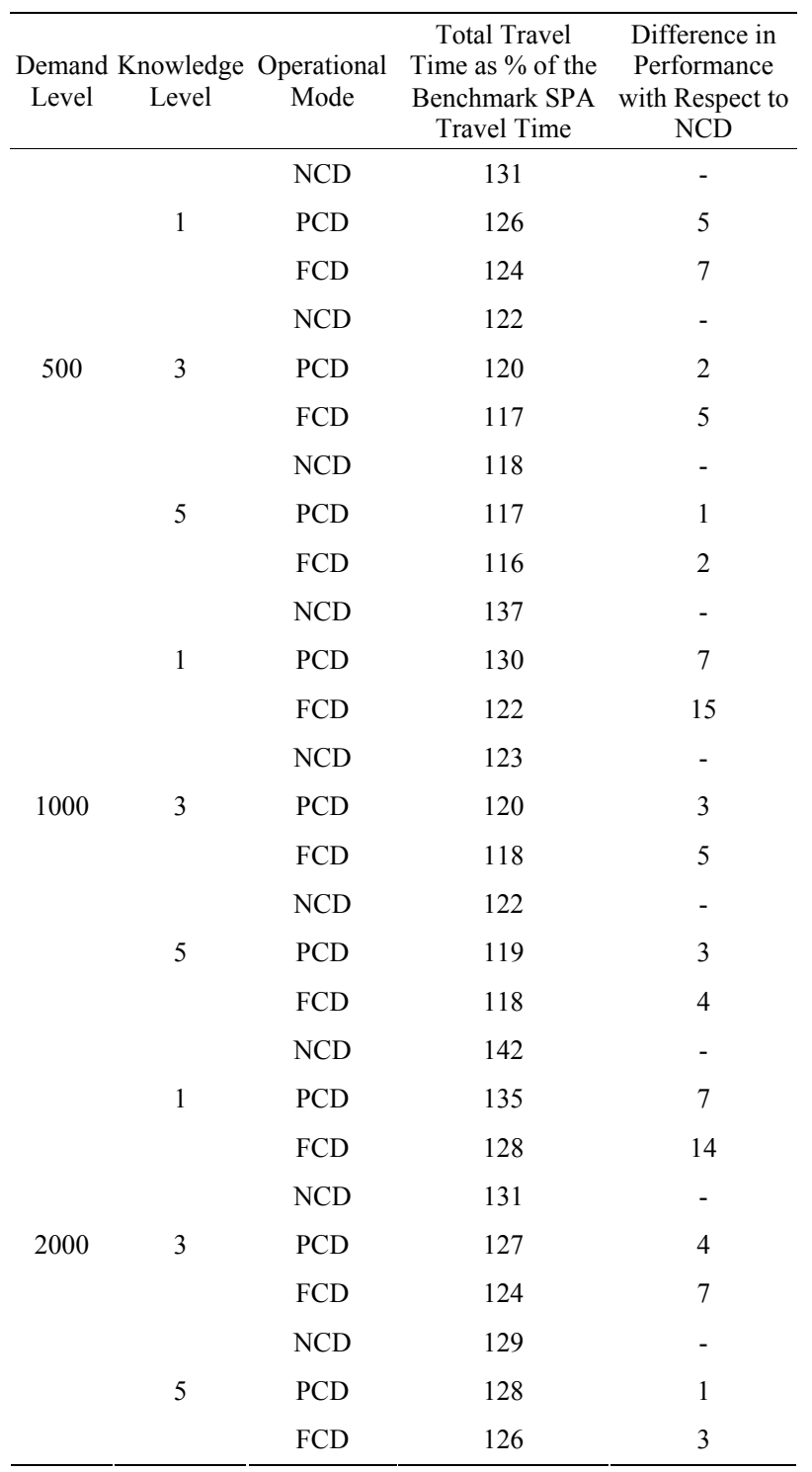

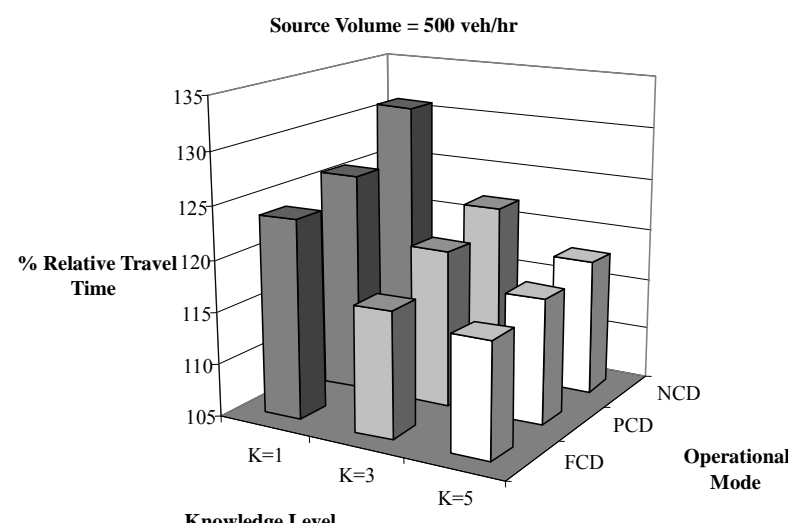

(a)

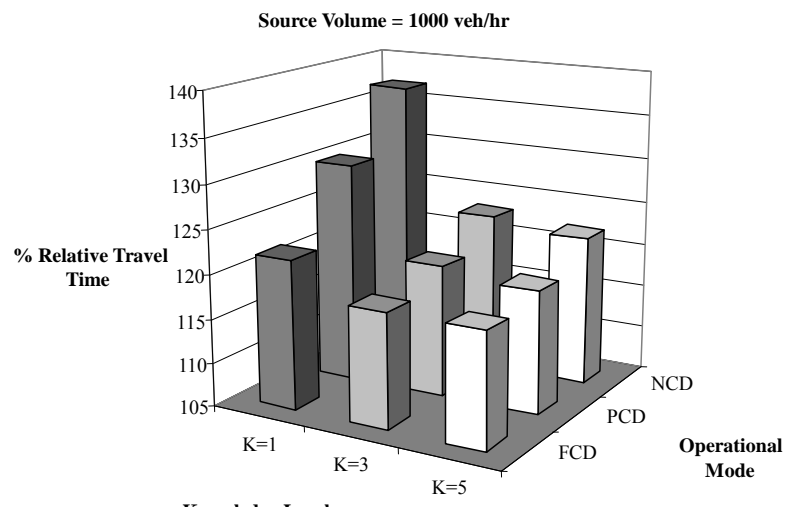

Knowledge Level

(b)

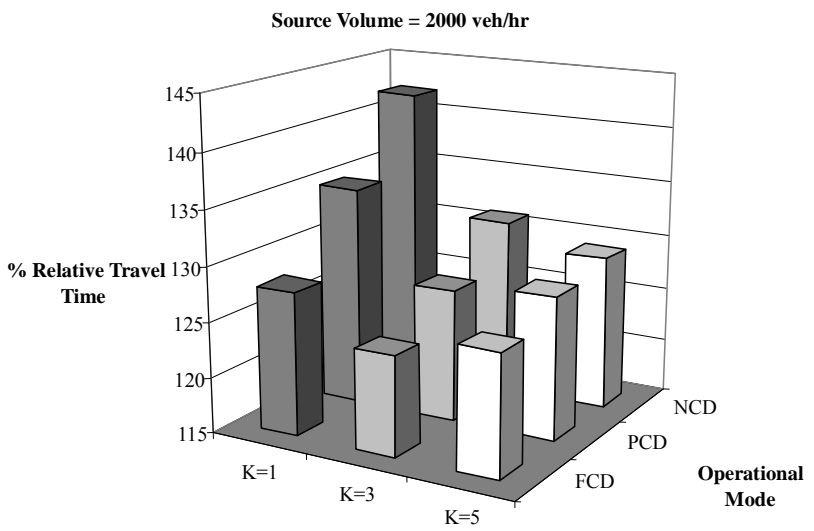

Knowledge Level

(c)

Figure 7. Performance of various cooperative schemes under various knowledge levels and (a) Low; (b) Medium and (c) High traffic intensities.

$2000 \mathrm{veh} / \mathrm{hr}$, and the three operational modes (FCD, $P C D$, and NCD). Table 2 shows the performance of the various operational modes in relative terms of the corresponding benchmark performance.

The results indicate that under low congestion levels ( $500 \mathrm{veh} / \mathrm{hr}$ ), the relative performance of the $F C D$ mode ranges from $116 \%$ to $124 \%$, and that makes this cooperative mode even more competitive with the centralized 
SPA scheme.

Under the $500 \mathrm{veh} / \mathrm{hr}$ scenario, the FCD mode resulted in about $7 \%$ reduction in overall travel time with knowledge level of $1,5 \%$ with knowledge level of 3 , and only $2 \%$ with knowledge level of 5 when compared to the non-cooperative scheme NCD. Under highly congested situations $(2000 \mathrm{veh} / \mathrm{hr})$, the reductions in overall travel time are $14 \%, 7 \%$ and $3 \%$ for knowledge levels of 1,3 and 5, respectively. The relative performance of the FCD mode ranges from $126 \%$ to $128 \%$.

The effect of adding $K_{i, j, \ell}^{L, t}$ to the heuristic function (between the FCD and PCD schemes) is notable at low knowledge and/or low congestion levels. At high knowledge levels, the concentration information, $K_{i, j, \ell}^{L, t}$, appears to have a limited impact on performance, and the local concentration variable, $K_{i, j, \ell}^{L, t}$, becomes adequate to capture the marginal cost.

The higher the knowledge level, the lesser the improvement (compared to $N C D$ ) achieved with the partial information scheme, $P C D$. Under limited knowledge levels, system performance can be enhanced by exchanged information. At high knowledge levels, the time lag assumed in transferring knowledge affects the accuracy of heuristic function estimates as compared to actually experienced values, and situations where the $P C D$ mode, or even the FCD mode, are worse than NCD could be observed.

To conclude, it is evident that the fully cooperative mode, $F C D$, exhibited a highly competitive performance compared to the centralized SPA scheme. The gain in performance achieved by the cooperative scheme declines with higher knowledge levels. The enhancement in performance under low knowledge levels, at which the non-cooperative scheme seems less effective, is quite significant. By achieving significant enhancement in performance under low knowledge levels, with negligible computational cost, the decentralized cooperative system becomes a very appealing route guidance system.

\section{Concluding Comments}

This paper presented a decentralized architecture that can be employed as a cooperative scheme for real-time route guidance in urban traffic networks. Simulation experiments were performed to assess the effectiveness of the proposed architecture. Under the cooperative scheme, the performance under low knowledge levels, at which the non-cooperative scheme seems less effective, is highly competitive with the SPA. By achieving significant enhancement in performance under low knowledge levels, with negligible computational cost, the decentralized cooperative system becomes a very appealing route guidance system with good potential for early deployment.

Bearing in mind the massive data processing and high operational cost associated with the centralized systems, the instability and communication constraints associated with the IVC-based systems, this paper seeks to provide improvement to the pure non-cooperative decentralized systems. The improvement is intended to resolve the reported cycling problems commonly encountered in the typical pure distributed systems. The improvement is sought through allowing for information exchange (or cooperation) among the various decentralized controllers. In a sense, we investigate the possibility of using intercontroller communication for exchanging knowledge regarding the traffic conditions in their respective territories. Such improvement is thought of as a way to overcome the limitations of the rapid topology changes, the frequent fragmentation and poor communication associated with the IVC-based systems, as well as the limitations of the heavy processing and cost of the centralized systems.

Further work will include the comparison of the presented system against the IVC-based route guidance systems reported in Hawas et al. (2009). It is expected that the two systems may exhibit similar performance as compared to the centralized system. Nonetheless, the cooperative decentralized system is expected to add no significant burden on the communication network. That is, in this case the IVC-based system, each vehicle communicates with many other vehicles, and this implies heavy communication and inter-vehicle processing requirements. In the case of the cooperative decentralized system the communication requirements are heavily reduced as each vehicle communicates only with one controller at a time. The fact that this communication is local with short distances allows for cheap communication media to be utilized.

\section{REFERENCES}

[1] J. L. Adler, et al., "A Multi Agent Approach to Cooperative Traffic Management and Route Guidance," Transportation Research Part B, Vol. 39, No. 4, 2005, pp. 297 318. doi:10.1016/j.trb.2004.03.005

[2] J. Anda, et al., "VGrid: Vehicular ad Hoc Networking and Computing Grid for Intelligent Traffic Control," Proceedings of the IEEE 61st Vehicular Technology Conference, vol. Vol. 61, No. 5, 2005, pp. 2905-2909.

[3] L. Chen, et al., "VGITS: ITS Based on Intervehicle Communication Networks and Grid Technology," Journal of Network and Computer Applications, Vol. 31, No. 3, 2007, pp. 285-302. doi:10.1016/j.jnca.2006.11.002

[4] Y .E. Hawas, "A Microscopic Simulation Model for Incident Modeling in Urban Networks," Transportation Planning and Technology, Vol. 30, No. 2, 2007, pp. 289309. doi:10.1080/03081060701398117

[5] Y. E. Hawas and M. Abdel Hameed, "A Multi-Stage Procedure for Validating Microscopic Traffic Simulation Models," Journal of Transportation Planning and Technology, Vol. 32, No. 1, 2009, pp. 71-91. 


\section{doi:10.1080/03081060902750686}

[6] Y. E. Hawas and H. S. Mahmassani, "Comparative Analysis of Robustness of Centralized and Distributed Network Route Control Systems in Incident Situations," Transportation Research Record, Vol. 1537, 1996, pp. 83-90. doi:10.3141/1537-12

[7] Y. E. Hawas, et al., "Comparative Assessment of Inter-Vehicular Communication (IVC) Algorithms for RealTime Traffic Route Guidance," Journal of Intelligent Transportation Systems: Technology, Planning and Operations, Vol. 13, No. 4, 2009, pp. 199-217. doi:10.1080/15472450903323107

[8] J. Jeremy, et al., "Challenges of Intervehicle ad Hoc Networks," IEEE Transaction on Intelligent Transportation System, Vol. 5, No. 4, 2004, pp. 347-351. doi:10.1109/TITS.2004.838218

[9] C. J. Jiang, et al., "Research on Traffic Information Grid," Journal of Computer Search and Development, Vol. 40, No. 12, 2003, pp. 1676-1681.

[10] H. S. Mahmassani and S. Peeta, "System Optimal Dynamic Assignment for Electronic Route Guidance in a Congested Traffic Network," Springer-Verlag, Heidel- berg, 1995.

[11] H. S. Mahmassani and Y. E. Hawas, "Experiments with a Rolling Horizon Dynamic Route Guidance Algorithm: Robustness under Stochastic Demands," Paper Presented at the INFORMS, Atlanta, 1996.

[12] T. Nadeem, et al., "Traffic View: A Scalable Traffic Monitoring System," Proceedings of the 2004 IEEE International Conference on Mobile Data Management, Berkeley, 19-22 January 2004, pp. 13-26.

[13] M. Papageorgiou, "Dynamic Modeling, Assignment and Route Guidance in Traffic Networks," Transportation Research, Vol. 24, No. 6, 1990, pp. 471-495.

[14] J. Pearl, "HEURISTICS: Intelligent Search Strategies for Computer Problem Solving," Addison-Wesley Publishing Co. Inc., Boston, 1984.

[15] P. E .Sarachick and U. Ozguner, "On Decentralized Dynamic Routing for Congested Traffic Networks," Transactions on Automatic Control, Vol. 27, No. 6, 1982, pp. 1233-1238.

[16] L. Wischhof, et al., "Information Dissemination in Self Organizing Intervehicle Networks," IEEE Transactions on ITS, Vol. 6, No. 1, 2005, pp. 90-101. 